\title{
UNA MIRADA A LOS SISTEMAS DE SALUD MEXICANO Y ARGENTINO.
}

\author{
A LOOK TO MEXICAN AND ARGENTINIAN \\ HEALTH SYSTEMS .
}

\section{UM OLHAR PARA OS SISTEMAS DE SAÚDE MEXICANOS E ARGENTINOS}

Trabajo de estudio bibliográfico y descriptivo resultante del Intercambio de estudiantes de posgrado Maestría en Enfermería de la Escuela de Enfermería y Obstetricia de la Universidad Nacional Autónoma de México, durante el mes de noviembre del 2018, en la sede de la Escuela de Salud Pública y Ambiente de la FCM Universidad Nacional de Córdoba

\section{Resumen}

Rosa Amarilis Zárate

Grajales ${ }^{1}$,

*Zoila Romualdo Pérez,

*Lucrecia Guadalupe

Sánchez Bañuelos,

*Noemí García Hernández,

*Anayeli Villegas Zúñiga,

*Diana Lizbeth Alvarado

Celaya.

${ }^{1}$ Profesor de T.C. Directora de la Escuela Nacional de

Enfermería y Obstetricia

Universidad Nacional

Autónoma de México. UNAM.

Camino Viejo a Xochimilco y

Viaducto Tlalpan s/n Col. San

Lorenzo Huipilco. Ciudad de

México. Tel. +5254851333,

Ext.201, E-mail. zarate

amarilis@hotmail.com

*Estudiantes del Programa de Maestría en Enfermería. UNAM.

Trabajo recibido: 10 de diciembre 2018.

Aprobado: 10 de marzo
En esta monografía se realiza una descripción del sistema de salud mexicano y argentino, destacando su estructura organizacional, para ello se describe el tipo de usuario, financiamiento, proveedores y compradores (instituciones de salud) de acuerdo a la organización del sistema de cada país. La monografía está estructurada en cuatro apartados: el primero comprende los antecedentes históricos, el segundo describe los sistemas de salud públicos en el que se menciona a la Secretaría de Salud de México y al Ministerio de Salud de Argentina; en el tercero el seguro social y en cuarto lugar se describe al sector privado de ambos países.En cada apartado se realiza una breve reflexión, en el que se concluye que en a pesar de la similitud del sistema económico de ambos países, existe una gran diferencia en la organización del sistema de salud.

Palabras clave: Sistemas de salud, seguridad social.

\section{Abstract}

In this work we describe the Mexican and Argentinian health systems, focusing on their organizational structure. Thus, type of users, funding, suppliers and buyers (health institutions) are described according to system organization in each country. The study is made 
up of four sections: the first one presents historical background, the second describes public health systems and the Health Secretary of Mexico and the Ministry of Health of Argentina are mentioned; the third section refers to social security; and the fourth describes the private sector in both countries. In each section there is a short reflection and the conclusion is that in spite of the similarities in the economic systems of both countries; there is a great difference regarding the organization of health systems.

Key words: Health systems, Social security, Public health.

\section{Resumo}

Nesta monografia é feita uma descrição do sistema de saúde mexicano e argentino, enfatizando a sua estrutura organizacional, para esse fim o tipo de usuário, financiamento, fornecedores e clientes (instituições de saúde) é descrito de acordo com a organização do sistema de cada país. A monografia é estruturada em quatro secções: a primeira abrange o pano de fundo histórico, a segunda descreve os sistemas de saúde pública nos quais o Ministério da Saúde do México e o Ministério da Saúde da Argentina são nomeados; no terceiro, o seguro social e em quarto lugar, descreve-se o setor privado de ambos os países. Em cada secção é feita uma breve reflexão, na qual se conclui que, apesar da similaridade do sistema econômico de ambos os países, há uma grande diferença na organização do sistema de saúde.

Palavras chave: Sistemas de saúde, seguridade social, saúde pública.

\section{Introducción}

México y Argentina son dos países de América Latina cuyos sistemas de salud se encuentran fragmentados(1) y a pesar de que difieren en su estructura organizacional, en ambos países se pueden distinguir tres sectores encargados de brindar servicios de atención a la salud: público, seguridad social y privado.

Cada país tiene sus propias características económicas, políticas y culturales por lo tanto la forma y tipo de servicios prestados, la distribución de recursos, el número de beneficiarios, la calidad de la atención, la eficiencia y eficacia de los sistemas a la población son distintas. En este sentido, se describe el sistema de salud de ambos países, desde una mirada reflexiva y crítica de algunos estudiantes de Maestría en Enfermería de la Universidad Nacional Autónoma de México (UNAM), en el marco de las prácticas profesionales en sistemas de salud, con sede en la Universidad Nacional de Córdoba (UNC), Argentina; en el cual se llevaron a cabo actividades académicas teóricas sobre el Sistema de Salud Argentino, actividades prácticas y de observación en un Centro de Salud dependiente de la universidad, en un hospital público y en uno privado. En relación al conocimiento del sistema de salud mexicano, la mayoría de los estudiantes son trabajadores tanto del sector público como del privado sumado a una previa revisión bibliográfica durante uno de los seminarios del programa de maestría.

El propósito de esta monografía es dar a conocer la mirada reflexiva con respecto a los sistemas de salud de los estudiantes del Programa de Maestría de Enfermería, de la UNAM con orientación en Administración del cuidado, el contenido del documento cuenta con la asesoría de la profesora responsable de la estancia académica.

La importancia de la información presentada en esta monografía, es relevante, ya que destaca los principales aspectos estructurales en ambos sistemas de salud, así como una descripción de aquellas similitudes o diferencias encontradas entre los dos países; dando al lector un panorama concreto del cómo viven mexicanos y argentinos el cuidado de su salud. 


\section{Antecedentes del sistema de salud argentino y mexicano.}

En los años de 1862 y 1946 la salud, no formaba parte de las funciones y obligaciones del Estado, sino que el servicio de atención a la salud de la población estaba a cargo por sociedades filantrópicas. No fue hasta el gobierno peronista (1946-1955) que se creó el Ministerio de Salud, cuando en la Conferencia internacional de la salud de Nueva York se estableció como obligatorio el derecho a la salud; a razón de ello el Estado asume la responsabilidad de proveer servicios de salud a todas las personas hecho que resultó en el desplazamiento de las sociedades filantrópicas.

Posteriormente en 1950, en la Confederación General del Trabajo se organizó un movimiento sindical que dio el surgimiento de las Obras Sociales (OS) a los cuales no era obligatorio afiliarse. Más tarde en el periodo de 1955 a 1983, el sector salud tuvo tres momentos históricos importantes el cual dio lugar a la situación actual del sistema de salud argentino. Dichos momentos fueron: la descentralización de los servicios estatales, aumento de la cobertura de los OS y el desarrollo del sector privado.

En el año de 1970, la afiliación a las OS eran obligatorias logrando cubrir el 75\% de la población, sin embargo, la cantidad de usuarios era excesivo en ese entonces y las obras sociales no podían cubrirlos en su totalidad, por tal razón las OS empezaron a contratar servicios del sector privado. A finales de este mismo año el estado disminuye su contribución a la función pública por consiguiente ocurrió la descentralización de los establecimientos sanitarios nacionales a instancias provinciales y municipales; de esta manera se traspasan los servicios de salud a la actividad privada, bajo la figura de empresas de medicina prepaga (EMC). Hasta el año de 1991 con la Reforma del Estado, el sector salud se dividió en tres: el sector público, Obras sociales y el privado. Las OS y el sector privado tienen la obligación de prestar como mínimo un plan de salud llamado Programa Médico Obligatorio (PMO)(1-4)

Por su parte, en México las grandes epidemias de viruela, tifus, el cólera y la malaria, así como problemas de mala alimentación, tuberculosis, el raquitismo y la peste durante el siglo XVIII, llevaron al sistema socio-político a reflexionar sobre la gravedad de los problemas sanitarios porque hasta ese entonces la prestación de los servicios de salud corría a cargo de órdenes religiosas, proveedores privados de medicina alopática y tradicional y de los servicios municipales de salud; por lo que el Real Tribunal del Protomedicato asume la responsabilidad de todos los problemas de salubridad, sobre todo en los casos de epidemias. Sin embargo, para 1831 el Presidente de la República, Anastasio Bustamante suprimió dicho tribunal y en su lugar estableció una junta denominada Facultad Médica del Distrito Federal con la obligación de elaborar un Código sanitario para el país(2)

Hasta el año de 1843, todavía no se concebía el derecho a la atención de la salud como un derecho autónomo, sólo se otorgaban facultades a los ayuntamientos para dar servicios de salud a la población. En 1859 el Estado mexicano se convirtió en el único encargado de velar por el cuidado y supervisión de la salud de sus habitantes, a través de la creación del Consejo Superior de Salubridad con el presidente Benito Juárez. Fue hasta el año de 1861 que el gobierno transfirió la responsabilidad de la prestación de los servicios de salud a los estados.

Durante la dictadura del general Porfirio Díaz (1876-1910), el sistema de salud se centralizó en la ciudad de México. No obstante, tras la segunda guerra mundial, surgió la necesidad de reorganizar el sector salud; por lo que, en 1943, la Dirección de Servicios Sociales, que había estado a cargo de los servicios hospitalarios desde 1877, se fusionó con el Departamento de Salubridad Pública dando lugar a la Secretaría de Salubridad y Asistencia (SSA). Posteriormente, el origen del sistema de seguridad social de México se remonta al artículo 123 de la Constitución de 1917, en donde se definen los derechos del trabajador. En este sentido, en 1943, se creó el Instituto Mexicano del Seguro Social (IMSS); posteriormente, el Instituto de Seguridad Social y Salud para los Trabajadores del Estado (ISSSTE), Petróleos Mexicanos (PEMEX). En 1979 las Fuerzas Armadas 
establecieron su propio servicio de salud. Todos los sistemas de seguridad social cubren al trabajador y a su familia, aunque los servicios que ofrecen son diferentes(5). En 1983 la protección de la salud de la población adoptó un rango constitucional al reformarse el artículo $4^{\circ}$, del cual surge la Ley General en Salud, que hasta la fecha dispone que toda persona tiene derecho a la protección de la salud(2)

Respecto al sector privado, históricamente antes del surgimiento de las instituciones públicas de salud, el cuidado de la salud de los mexicanos estaba a cargo de los profesionales que se dedicaban a la práctica privada. Sin embargo, por la creación de leyes y reformas, el sector público alcanzó mayor porcentaje de cobertura para la atención a la salud, sin embargo, dadas las deficiencias del sistema público de salud y la falta de inversión a la misma, el sector privado tuvo una aportación importante en la preservación de la salud. Según información generada por el propio gobierno, para principios de la década de 20002010 , más del $50 \%$ del gasto total en salud provenía del gasto privado, y de éste más del 90\% estaba relacionado con el gasto personal de bolsillo; desde entonces el sector privado tiene sus propios servicios de atención a la salud(6).

Por necesidad sanitaria de la población de ambos países, sus gobiernos estructuraron un sistema de salud con el objetivo de mejorar las condiciones de vida de la población logrando establecer como la salud como un derecho; sin embargo, dadas las características de fragmentación del sistema de salud de ambos países, en el presente no todos gozan de dichos derechos, sobre todo aquellas personas que viven en condiciones geográficas marginadas y pobreza. Bajo este panorama, el gobierno mexicano tiene en sus proyectos crear un único sistema de atención a la salud el cual no ha sido posible debido a cuestiones políticas y económicas que desde la historia siguen sellando la situación del país. Por su parte Argentina, ha tenido mayor control en el acceso a la salud debido a la participación del sector privado. Después de esta breve revisión histórica, a continuación, se presenta la descripción del funcionamiento y organización del actual sistema de salud de cada país, en cada apartado se incluyen algunas reflexiones y críticas de los autores de la presente monografía desde su mirada como estudiantes de maestría del área de la salud.

\section{Sistema de salud público}

Antes que nada, es necesario aclarar la característica estructural del sistema de salud de cada país porque en la medida en que son muy similares hay una gran diferencia en su organización, explico. En México existen dos sectores de salud, el público y el privado; en Argentina hay tres el público, seguridad social y el privado. La diferencia está en que en México el sector público está conformado por los Servicios Estatales de Salud (SESA) y la Secretaría de Salud (SSA) y por la Seguridad social, mientras que en Argentina el sector público lo conforma solo el Ministerio de Salud Nacional y de las provincias. En este apartado no se abordará la seguridad social de México, aunque pertenezca al sector público con el fin de mantener coherencia en el contenido de la presente monografía.

Ahora bien, el sector de salud público de los argentinos está integrado por estructuras administrativas de nivel nacional, provincial y municipal, cuyos proveedores son la red de hospitales y centros de salud públicos y el personal que ahí labora(1), quienes prestan atención gratuita a toda la población que lo requiera, sobre todo a personas que no tienen acceso a la seguridad social(7) y además no disponen de suficientes recursos financieros para acudir a instalaciones privadas $(1,8)$; la situación es similar en el contexto mexicano ya que son los auto-empleados, trabajadores del sector informal y desempleados quienes utilizan los servicios públicos.

Desde el punto de vista institucional, en Argentina el Ministerio de Salud es la máxima autoridad nacional en materia de salud(9), mientras que en México es la Secretaría de Salud(SSA) quien se encarga primordialmente de la prevención de enfermedades y la promoción de la salud(10) y regula el funcionamiento efectivo y eficiente de todos los organismos de salud existentes en el país; mientras que en Argentina en el ámbito del 
gobierno nacional, funciona la Superintendencia de Servicios de salud, el cual tiene funciones similares a la Secretaría de Salud de México, tiene por misión regular, controlar y asegurar el cumplimiento las políticas de promoción, reservación y recuperación de la salud de la población y la efectiva realización del derecho a gozar las prestaciones de salud establecidas en la legislación vigente(11).

En cuanto al financiamiento en México, la SSA y el SESA obtienen sus fondos a través de la contribución del gobierno federal y de la contribución de los gobiernos estatales, debido a esta situación en el 2003 la SSA impulsó el Seguro Popular de la Salud (SPS), con la con la finalidad de mejorar la cobertura en salud(12) para el cual se destinó el $2.7 \%$ del PIB(13, 14), el financiamiento de dicho seguro es tripartita: aportes del gobierno federal y del estatal y la cuota de recuperación que corresponde al 15\% del salario mínimo de cotización misma que es cubierta por el gobierno nacional y los aportes de sus afiliados a través de la Cuota Familiar, cabe aclarar el en la actualidad el SPS no ha tenido mayor impacto en la salud pública. Por su parte, el sistema de salud argentino obtiene su financiamiento de los recursos nacionales, provinciales y municipales, además, recibe pagos ocasionales de parte del sistema de seguridad social cuando atiende a sus afiliados. En ambos países se destina un presupuesto a cada estado o provincia y es responsabilidad del mismo la forma en que se administra; en Argentina en 2017 se destinó el 2.19\% de PIB para el gasto en salud pública(15) mientras que en México, que por cierto se ubica en el último lugar de los países miembros de la OCDE en cuanto al gasto en salud como porcentaje del PIB, para el año 2017 se destinó el 2.7\% del PIB al gasto en salud, cuando el promedio de los países de la OCDE es 6.6\%(16), de modo que en ambos países el PIB destinado fue muy por debajo de la media.

En cuanto a los compradores, es decir, las instituciones de salud que prestan los servicios públicos, en México es la secretaría de salud y el SESA, mismos que tienen distribuidas clínicas, centros de salud y hospitales en todos los estados y sus respectivas regiones. Así mismo Argentina, cuenta con una red pública de servicios sanitarios distribuidos en las provincias y municipios, sin embargo dada la estructura federal, los gobiernos provinciales tienen la mayor parte de las responsabilidades en la provisión de servicios, lo que hace que las provincias sean autónomas en sus decisiones y los lineamientos nacionales solo tengan un valor indicativo, a diferencia de México la Secretaría Salud es la que regula todas las instalaciones que otorguen servicios de salud en el país.

En relación al párrafo anterior, como estrategia anta la alta demanda de salud de la población abierta (población no asegurada) el gobierno mexicano creó IMSS Prospera, el cual es un programa que contribuye a garantizar la protección de la salud, mediante el otorgamiento de servicios preventivos gratuitos en comunidades rurales y urbanas marginadas del país(17) y en coordinación son la Secretaría de Salud brindan atención al $60 \%$ del total de la población mexicana(12,18). Por su parte el Ministerio de Salud de Argentina comparte con otras instancias del gobierno nacional varios programas sociales. En ambos países el sector público tiene la intención de brindar una cobertura universal en salud, sin embargo, las brechas políticas, económicas y sociales no han permitido consolidar tan fin; situación que es similar en la gran mayoría de los países de América Latina.

\section{Seguridad social}

La Constitución Política de la República Mexicana del año 1857, es donde se establecen los primeros derechos de los trabajadores, sin embargo, en la Constitución de Política de 1917, se reivindicaron los derechos laborales al introducir más beneficios para los trabajadores, comola responsabilidad de los patrones en los accidentes de trabajo y enfermedades, la obligación de vigilar los mandatos legales sobre la higiene, seguridad y la previsión popular $(19,20)$. La Seguridad social es un término que se refiere al bienestar de los ciudadanos y se encuentra encaminada a la protección y mejoramiento de los niveles 
en bienestar de las personas trabajadoras y sus familias (21).

En Argentina la salud es un derecho colectivo, público y social de seguridad constitucional, anclado en el art. 42 de la Constitución Nacional $(15,22)$, donde se establece que "los consumidores y usuarios de bienes y servicios tienen derecho, en la relación de consumo, a la protección de su salud, seguridad e intereses económicos; a una información adecuada y veraz; a la libertad de elección, y a condiciones de trato equitativo y digno" (23).

La fuente financiera de la seguridad social en ambos países es de tipo tripartita, ya que se da la contribución del trabajador, del empleador y gubernamental de tipo nacional o estatal en el caso mexicano, o provinciales y nacionales en el caso argentino $(14,24)$.

La seguridad social se encuentra dentro del sector público del sistema de salud mexicano; del cual los compradores son: el Instituto Mexicano del Seguro Social (IMSS), Instituto de Seguridad y Servicios Sociales de los Trabajadores del Estado (ISSSTE), Petróleos Mexicanos (PEMEX), Secretaría de la Defensa (SEDENA) y Secretaría de Marina (SEMAR) (14). Mientras que en Argentina el sistema de salud se integra por tres sectores: público, de seguridad social y privado. En el sector de la seguridad social, se integran las Obras Sociales (OS) las cuales pueden ser nacionales o provinciales $(15,22)$.

En Argentina el sector del seguro social obligatorio está organizado en torno a las Obras Sociales (OS), que aseguran y prestan servicios a los trabajadores y sus familias. La mayoría de las OS operan a través de contratos con prestadores privados y obtienen el financiamiento de contribuciones de los trabajadores y patronales $(24,25)$.

El financiamiento de las OS nacionales proviene del pago de $8 \%$ del salario de los trabajadores activos; $3 \%$ lo aporta el trabajador y 5\% el patrón. Las OS provinciales se financian de la misma manera, con contribuciones de los empleados gubernamentales y con contribuciones de los gobiernos provinciales en su calidad de empleadores(24).

En México, el gasto total en salud como porcentaje del PIB aumentó de 5.1\% en 2000 a $5.9 \%$ en 2008 y el gasto público en salud representó $46.7 \%$ del gasto total en salud. En 2007, el gasto privado en salud concentró 54.6\% del gasto total en salud. Alrededor de $93 \%$ de este gasto son pagos de bolsillo y $7 \%$ corresponde al pago de primas de seguros médicos privados. En Argentina, Brasil, Colombia y Uruguay el gasto de bolsillo concentra 41, 64, 42 y 31\% del gasto privado. México presenta, por lo tanto, una de las cifras más altas de gasto de bolsillo de la región. Este gasto expone a las familias a gastos catastróficos y/o empobrecedores (14).

En cuanto a los proveedores de este servicio existen diferencias entre ambos países, ya que en México son hospitales, clínicas y médicos pertenecientes a las instituciones IMSS, ISSSTE, PEMEX, SEDENA, MARINA quienes brindan la atención a los trabajadores del sector formal, familias de los trabajadores y los jubilados; a diferencia que en Argentina los proveedores son hospitales y centros de salud públicos, y empresas de medicina prepaga quienes prestan su servicio a personas beneficiarias, pensiones no contributivas, trabajadores y sus beneficiarios y jubilados.

En Argentina, el sector del seguro social obligatorio se organiza en torno a las Obras Sociales (OS), que cubren a los trabajadores asalariados y sus familias según ramas de actividad. Además, cada provincia cuenta con una OS que cubre a los empleados públicos de su jurisdicción. Finalmente, el Instituto Nacional de Servicios Sociales para Jubilados y Pensionados/Programa de Asistencia Médica Integral (INSSJyP - PAMI) brinda cobertura a Prepaga, que incluyen un subsector prestador de servicios agrupado en la confederación Argentina de Clínicas, Sanatorios y Hospitales Privados. Este sector comprende también a las llamadas cooperativas y mutuales de salud, que son entidades no lucrativas que ofrecen planes de salud pero no operan ni como OS ni como medicina prepagada(24).

Las OS nacionales y las Empresas de Medicina Prepaga (EMP) tienen la obligación de atender el Programa Médico Obligatorio (PMO) dispuesto por el Ministerio de Salud, cuyo cumplimiento es supervisado por la Superintendencia de Servicios de Salud (SSS) dependiente de dicho ministerio. El PMO comprende un conjunto de prestaciones muy 
amplio y los medicamentos respectivos. Este programa cubre $95 \%$ de las causas de consulta ambulatoria, atención quirúrgica y hospitalaria, atención odontológica, salud mental, rehabilitación y cuidados paliativos(24,25,26).Al igual que en Argentina, en México las instituciones prestan los servicios de salud a sus beneficiarios en función de su condición laboral. Los trabajadores del sector formal de la economía, activos y jubilados, y sus familias son los beneficiarios de las instituciones de seguridad social, que cubren a 48.3 millones de personas. El IMSS cubre a más de $80 \%$ de esta población y la atiende en sus propias unidades con sus propios médicos y enfermeras. El ISSSTE da cobertura a otro $18 \%$ de la población asegurada, también en unidades y con recursos humanos propios. Por su parte, los servicios médicos para los empleados de PEMEX, SEDENA y SEMAR en conjunto se encargan de proveer de servicios de salud a $1 \%$ de la población con seguridad social en clínicas y hospitales de PEMEX y las Fuerzas Armadas, y con médicos y enfermeras propios.

De acuerdo a los elementos antes mencionados de la seguridad social en México y Argentina, se puede visualizar las diferencias en su estructura dentro del sector, compradores y proveedores, ya que se encuentran organizados de manera diferente ya que en el caso de Argentina el apoyo es de los hospitales y clínicas del sector público y privado para atender a su población, mientras que México cuenta con instituciones para este fin que son independientes de los públicos y privados. Pero también podemos observar que su financiamiento y los usuarios son iguales.

\section{Servicios de atención privada}

El sector privado comprende a los prestadores de servicios que trabajan en consultorios, clínicas y hospitales privados, incluyendo a los prestadores de servicios de medicina alternativa y a las compañías aseguradoras las cuales básicamente son de gastos médicos mayores que a su vez tienen sus propias redes de servicios, este sector ofrece sus servicios principalmente con fines de lucro(14).

El financiamiento de este sector deriva de los pagos directos de bolsillo de las personas con capacidad de pago al momento de recibir atención médica, adquirir medicamentos o insumos, además de los pagos anticipados correspondientes a las primas de los seguros de gastos médicos mayores(27).

Antes del surgimiento de las instituciones públicas de salud, el cuidado de la salud de los mexicanos estaba a cargo de los profesionales que se dedicaban a la práctica privada. Para principios de la década de 2000-2010, más del 50\% del gasto total en salud provenía del gasto privado, y de éste más del $90 \%$ estaba relacionado con el gasto personal de bolsillo, es decir los mexicanos se veían obligados a financiar la atención de su salud, incurriendo así en lo que se llama gastos catastróficos(6). Actualmente solo las personas que tienen ingresos económicos altos pueden acceder a estos servicios; sin embargo, existen empresas aseguradoras que se encargan de gestionar los gastos de salud de empleados quienes por lo regular laboran en una empresa privada.

Argentina por su parte, el ejercicio profesional privado se ha desarrollado paralelamente al desarrollo del país y a la capacidad de los habitantes para afrontar los gastos de atención médica, por lo tanto presta servicios a personas y familias que pagan por acto médico o que pagan las cuotas de un plan de salud (pre-pago), también está conformado por profesionales de la salud y establecimientos que atienden a demandantes individuales, a los beneficiarios de las obras sociales y de los seguros privados. También incluye entidades de seguro voluntario llamadas Empresas de Medicina Prepaga que se financian sobre todo con primas que pagan las familias y/o las empresas(24).

En 2013, en México se contabilizaron 2301 centros de atención privada en salud, en los cuales 1453 eran Instituciones Generales, 19 Psiquiátricos y de tratamiento de adicciones y 829 de Especialidades Médicas. Teniendo un ingreso por 47,023 millones de pesos, la Ciudad de México, Jalisco y el Estado de México concentraron el 30.8\%. En 2014, el 69\% 
de las unidades de hospitalización correspondían al sector privado, pero solamente el 29\% del total de camas, lo cual indica que los hospitales de este sector son pequeños al igual que la población a la que se atiende. Sin embargo, debido a la falta de calidad y oportunidad de los servicios otorgados en el sector público - los cuales en su mayoría se centran en la atención de la enfermedad a diferencia de sector privado cuyo centro de atención en la persona- en los últimos años ha incrementado la utilización de los servicios del sector privado aun cuando los usuarios estén afiliados a algunas de las instituciones públicas de salud. Considerando este contexto, las empresas de salud privadas han diversificado las opciones de atención, por ejemplo, "a partir de la regulación de la venta de antibióticos con prescripción médica en 2010, los consultorios médicos adyacentes a farmacias privadas han crecido hasta representar el 17\% del total nacional de consultas ambulatorias"(28).

Por su parte en Argentina, las entidades de medicina prepaga cubren una población cercana a 2,500,000 personas, de las cuales el $60 \%$ se afilia en forma individual o familiar, y el resto a través de empresas(15).Los prestadores privados son hoy los principales proveedores de servicios asistenciales para los beneficiarios de las obras sociales y por agrupamientos locales, provinciales y nacionales han constituido grandes organizaciones representativas de los prestadores de todo el país como la Confederación Argentina de Clínicas, Sanatorios y Hospitales Privados (CONFECLISA), Confederación Médica de la República Argentina (COMRA), Confederación Odontológica de la República Argentina (CORA), Confederación Unificada Bioquímica de la República Argentina (CUBRA).

Las empresas de Medicina Prepaga presentan mayor dispersión de precios y servicios cubiertos que el de las Obras Sociales, se agrupan en dos cámaras empresariales: la Cámara de instituciones Médico-Asistencial de la República Argentina y la Asociación de Entidades de Medicina Prepaga (que representa a las empresas sin servicios propios). Las cámaras agrupan a alrededor de 70 firmas de un total estimado de 150 con ubicación en Capital Federal y Conurbano y 46 empresas radicadas en el interior. Cabe aclarar que la afiliación se concentra fundamentalmente en la Capital Federal y, en menor medida, en el Gran Buenos Aires, existiendo algunas importantes entidades en las provincias de Santa Fe, Córdoba, Mendoza, Entre Ríos y Tucumán(29).

En consideración a los sectores privados de ambos países, se observa que el pago de los servicios involucra directamente gastos de bolsillo elevados para obtener un bien de salud, sin embargo, en ambos contextos se pueden acceder a los servicios gracias al pago de cuotas de un seguro, por lo tanto, realizando una comparación del gasto en salud en estos países, en México el PIB en salud es de 5.9\% y en Argentina es de 9.8\%. En 2007 en México el gasto privado en salud concentró $54.6 \%$ del gasto total, del cual el $93 \%$ son gastos de bolsillo y $7 \%$ deriva de los seguro médicos privados, mientras que en Argentina el gasto en salud fue del $41 \%$, del cual $44 \%$ pertenece al gasto de bolsillo y $56 \%$ se concentra en los seguros privados $(15-18,24,27)$. Por consiguiente, los mexicanos tienen mayor gasto de bolsillo lo que a su vez genera mayores dificultades para acceder a los servicios de salud.

En relación a lo anterior, el gasto realizado por las familias es elevado, sin embargo, en Argentina se observa una mayor cobertura del gasto por las aseguradoras a diferencia de México, en el cual el gasto realizado por las familias es mayor. Cabe mencionar, que el sector privado de Argentina también atiende a personas beneficiarias de las Obras Sociales, a diferencia de México, cuyos únicos beneficiarios son un grupo limitado de personas con capacidad de pago.

En cuanto a la distribución de instituciones privadas, en México hasta el 2013 se contabilizaron 2301, mientras que en Argentina existen alrededor de 150, datos que están directamente relacionados con la pirámide poblacional porque la República Mexicana cuenta con aproximadamente 127 millones de personas a diferencia de la República Argentina cuya población es de 44.27 millones de habitantes. No obstante, la concentración del sector privado se encuentra en la capital federal y en las grandes ciudades con mayor 
desarrollo económico. Cabe resaltar que cada uno de estos países cuenta con cuatro hospitales privados que se encuentran dentro del Ranking Mundial de Hospitales(30).

\section{Conclusiones}

Analizando los sistemas de salud de México y Argentina se observa la fragmentación de los servicios que otorgan, ambos cuentan con un sector público, seguridad social (obras sociales) y un sector privado. Sin embargo, en Argentina el acceso a cualquier unidad de salud en caso de emergencia es indiscriminado sin importar a que sistema pertenezca, situación que en México no sucede, pues solo se brinda atención gratuita dependiendo del sector al que pertenezca el derechohabiente. Sin embargo, si bien la población argentina en su conjunto tiene la posibilidad de acceder a los servicios ofrecidos por el sector público, aún persisten brechas importantes y asignaturas pendientes, especialmente en lo relativo a su calidad.

Es importante para ambos países fortalecer líneas enfocadas en la prevención y promoción de la salud, ya que, comparten las mismas problemáticas epidemiológicas, como lo son las enfermedades crónicas no transmisibles (Obesidad, Diabetes, Hipertensión), por ello, los programas que se implementen deben considerar las características específicas que distinguen a cada grupo de su población.

Cada uno de los sistemas debe continuar generando estrategias de acuerdo a las condiciones políticas, sociales, culturales y económicas de cada país, con el objetivo de lograr la cobertura universal y el acceso total a la salud.

\section{Bibliografía}

1. Giedion Ú, Villar M, Ávila A. Los Sistemas de Salud en Latinoamérica y el papel del Seguro Privado. España: Fundación MAPFRE; 2010.

2. Tobar F. Breve historia del sistema argentino de salud. In: Garay O, editor. Responsabilidad Profesional de los Médicos Ética, Bioética y Jurídica Civil y Penal. Buenos Aires: La Ley; 2012. p. 1-19.

3. Tobar F. El modelo de salud argentino: historia, características, fallas.: Ministerio de salud; 2017 [cited 03 de Diciembre 2018]. Available from: https://bit.ly/2Q87uE9.

4. Urbina P. Estado y salud en Argentina: Una aproximación histórica.: Ministerio de Salud y Desarrollo Social; 2017 [08 Marzo 2019]. Available from: https://bit. ly/2UnPgwd.

5. $\quad \mathrm{N} \mathrm{H}$, A U. 25 años de descentralización del sistema de salud mexicano:una experiencia para analizar2008 [22 Agosto 2018]; 7(15):[26-43 pp.]. Available from: https://bit.ly/2woLRCT.

6. B Z, T R. Desempeño del sector privado de la salud en México. FnSalud [Internet]. D [24 Agosto 2018]:[153-62 pp.]. Available from: https://bit.ly/2P8wlm6.

7. Litewka S. Quality, health system and governance. The argentinean case. 2010;16(2):148.

8. Arce H. Organización y financiamiento del sistema de salud en la Argentina. 2012 03 Diciembre 2018; 72(5):[414-8 pp.]. Available from: https://bit.ly/2riia3Z.

9. Centrángolo O, Devoto F. Organización de la salud en Argentina y equidad. Una reflexión sobre las reformas de los años noventa e impacto de la crisis actual: Comisión Económica para América Latina y el Caribe; 2002.

10. Gómez C. El sistema de Salud en México. Revista CONAMED [Internet]. 2017 03 de Diciembre 2018; 22(3):[129-35 pp.]. Available from: https://bit.ly/2BOt8Vh.

11. MinisteriodeSaludyDesarrolloSocial. Superintendencia de Servicios de Salud [08 Marzo 2019]. Available from: https://bit.ly/2SOoko1.

12. InstitutoNacionaldeEstadísticayGeografía. Encuesta Intercensal 2015: Principales 
resultados 2015 [12 Febrero 2019]. Available from: https://bit.ly/2E6Kilq.

13. OECDHealthStatistics. [03 Diciembre 2018]. Available from: https://bit.ly/2rlWGDi.

14. Dantés O, Sesma S, Becerril V. Sistema de salud de México. Salud pública Méx. 2011(53):s220-s32.

15. Galli A, Fagés M, Swieszkowski S. El Sistema de Salud Argentino: Sociedad Argentina de Cardiología; 2017 [03 Diciembre 2018]. Available from: https://bit.ly/2Ohp7jH.

16. Méndez J. Gasto en Salud propuesta 2017: CIEP; 2017.

17. López O, Jarillo E. La reforma neoliberal de un sistema de salud: evidencia del caso mexicano. Cad Saúde Pública. 2017;33 $\operatorname{Sup}(2 \mathrm{e}): 1-13$.

18. InstitutoMexicanodelSeguroSocial. Programa IMSSPROSPERA 2016 [03 Diciembre 2018]. Available from: https://bit.ly/2UcNeQb.

19. Universidad Nacional Autónoma de México (UNAM). La seguridad social en México. Disponible en: https://bit. ly/2mhrgux

20. Narro J, Moctezuma D, Orozco L. Hacia un nuevo modelo de seguridad social. 2010;7(20):7-33.

21. Procuraduría General de la Defensa del Trabajo. La seguridad social y sus beneficios 2018 [03 Diciembre 2018]. Available from: https://bit.ly/2yY0OOn.

22. Ministerio de Salud. Derecho a la salud. Argentina. 2016 [04 Diciembre 2018]. Available from: https://bit. ly/2EcPQbR.

23. Donato N. Derecho a la salud. Ministerio de Salud Presidencia de la Nación. 2017[04 Diciembre 2018]. Disponible en: https://bit.ly/2Cl1vTw

24. Belló M, Becerril V, Knaul F, Arreola H, Frenk J. Sistema de salud de Argentina. Salud pública Méx 2011;53(2):s96-s109.

25. Spinelli H. Las dimensiones del campo de la salud en Argentina2010 [03 Diciembre 2018]; 6(3):[275-93 pp.]. Available from: https://bit.ly/2zHCT5X.

26. Tobar F, Olaviaga S, Solano R. Retos postergados y nuevos desafíos del sistema de salud argentino. 2011 [03 Diciembre 2018]. Available from: https://bit.ly/2RtG888.

27. BancoMundial. Sistema de Salud de México: Proyecto DIME; 2014 [03 Diciembre 2018]. Available from: https://bit.ly/2rjq9Oj.

28. SecretaríadeSalud. Informe sobre la salud de los mexicanos 2016. Diagnóstico general del sistema de salud 2016 [03 Diciembre 2018]. Available from: https:// bit.ly/2jLkJbk.

29. C M. Prestaciones médicas 2017 [03 Diciembre 2018]. Available from: https://bit.ly/2KQ59Ys.

30. LaboratoriodeCibermetría. Ranking Web Hospitales 2018 [03 Diciembre 2018]. Available from: https://bit. ly/2KQUUDb. 